\title{
Research Progress and Application of Computer Artificial Intelligence Technology
}

\author{
Jin Wei \\ Northwestern Polytechnical University Ming De College, Xi’an, China 710124
}

\begin{abstract}
With the development of science and technology, artificial intelligence technology has received more and more attention and attention. Under the background of the rapid development of big data and cloud computing, the artificial intelligence industry broke out. There is a huge amount of research on artificial intelligence and the artificial intelligence industry is huge. As far as the artificial intelligence industry in China is concerned, even the start is relatively late, but the industry scale, industrial layout, and technology research are all in a continuous improvement stage. Especially after the deepening of the layout of science and technology and manufacturing industries, the scale of artificial intelligence industry is further developed. More artificial intelligence products will appear at the same time. From the perspective of the concept, development history and new progress of artificial intelligence, this paper combines China's artificial intelligence market and the development of artificial intelligence companies to analyze the current major application areas, and then further explore the future development trend of artificial intelligence.
\end{abstract}

\section{The Concept, Development History and New Progress of Artificial Intelligence}

\subsection{The Concept of Artificial Intelligence}

Artificial intelligence technology is an important branch in the field of computers. It refers to the simulation based on computer simulation technology. It simulates human thinking and behavior patterns, so that computer hardware devices can have human-like response capabilities, making computer hardware intelligent on the surface. So as to provide people with corresponding intelligent services. Artificial intelligence technology is not only artificial intelligence robots, but also includes virtual reality, speech recognition, automatic processing, and computer neural networks. In its development, it is not a single development, but a process that interacts with and merges with other disciplines. This technology has high efficiency in processing data, which can effectively solve uncertainties, learn new knowledge, and perform calculations on complex problems and learn the processing features [1].

\subsection{Artificial Intelligence Development Process}

\subsubsection{The Rise of Artificial Intelligence}

The concept of artificial intelligence was first proposed around the 1950s. Afterwards, many researches on artificial intelligence began, and achievements such as proof of machine theorem and processing of LISP table processing languages were achieved. However, due to the rise of artificial intelligence at that time and the fact that the technology involved multiple disciplines and multiple technical fields, artificial intelligence was limited by inference algorithms, machine translations and so on. And in the early development period other technologies also developed slowly.

\subsubsection{Artificial Intelligence Technology Development Tide}

Since then, along with the development of other technologies and further research on artificial intelligence technology, experts and scholars in this field have developed an artificial intelligence expert system until the 1970s. The development of this system has promoted artificial intelligence technology into a new era. At the stage of development, research on artificial intelligence entered a period of high development.

\subsubsection{Major Development Period of Artificial Intelligence Technology}

With the development of computer technology, after the successful development of the fifth-generation computer, the artificial intelligence technology has also been further developed, and the study of this technology has entered a major period of development. This also shows that the research and development of artificial intelligence technology can not be separated from the development of computer technology, and the two are almost 
synchronized.

\subsection{New Progress in Artificial Intelligence Research}

In the information age, people's lives and work have been inseparable from computer networks and often require computers to complete information retrieval and other services. However, the problem that needs to be further solved in the computer field is how to obtain valuable information from complex information data, how to enable computer technology to provide people with real-time and convenient services, and how to make computer interfaces more humanized. These have become hot topics in artificial intelligence research. In addition, with the birth of cloud computing and big data, research on artificial intelligence is also moving toward data mining, knowledge realization, and intelligent interface technology.

\section{Artificial Intelligence Market Analysis and China's Artificial Intelligence Enterprise Analysis}

\section{Forecast of China's Artificial Intelligence Market Size in Year 2018-2021}

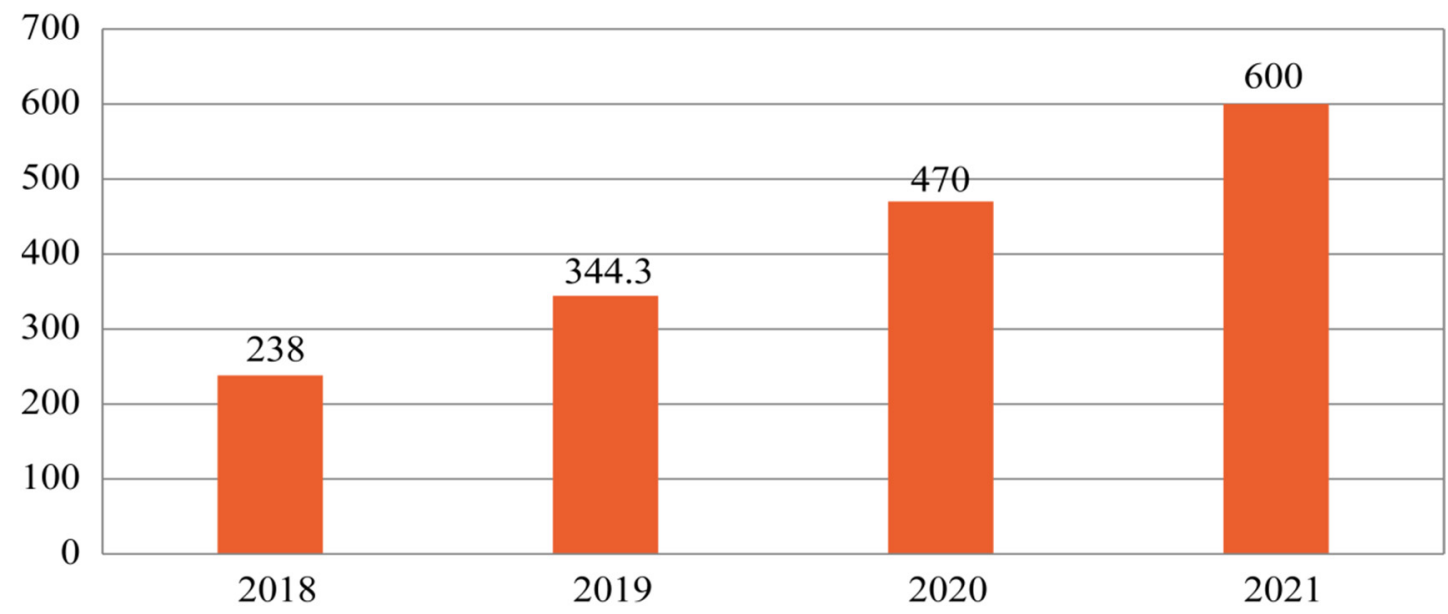

- Intelligence Market (Billion)

\subsection{China's Artificial Intelligence Enterprise Analysis}

The number of artificial intelligence startup companies in China has risen since 2014, and there has been no decrease in the entrepreneurial enthusiasm for artificial intelligence until 2016, indicating that the artificial intelligence market has great prospects. At the end of 2016, the number of artificial intelligence startup companies reached more than 200. The main two directions of startup companies are voice and vision, and there are also many artificial intelligence dedicated chip

\subsection{Artificial Intelligence Market Analysis}

2016 is the first year of artificial intelligence. Since then, the artificial intelligence industry has broken out with the support of cloud computing and big data. Various researches on artificial intelligence have appeared in people's horizons, and the future market space is also very large. As far as China is concerned, the scale of the artificial intelligence industry reached 10 billion in 2016 . The growth rate in 2017 exceeded 50\%, and the industrial scale exceeded 15 billion yuan. It is estimated that by 2019, the artificial intelligence market in China will reach 34.4 billion yuan. With the deepening of industrial layout and technological research, as well as the deepening of the layout of technology and manufacturing industries, the market size of artificial intelligence has further expanded, and the emergence of numerous artificial intelligence startup companies will also bring more artificial intelligence products. For the artificial intelligence investment, there were 48 institutions investing in 2015, with an investment of 1.423 billion yuan. It is estimated that by 2021, the market size of artificial intelligence will reach 60 billion yuan [2]. companies. Based on an analysis of non-listed companies that use artificial intelligence as their driving technology and business, it was found that most companies were established in 2015, followed by 2014 and 2016. Regarding the startup direction of artificial intelligence startups, the largest number is in the field of computer vision, followed by the field of service robotics and speech, and others include smart medical, machine learning, and smart driving. Among them, computer vision technology is an important core technology of artificial intelligence. It can be applied in fields such as security, finance, hardware, marketing, and driving. The level of technology development is basically close to the international level. 


\section{Distribution of China's artificial intelligence startup companies in 2016}

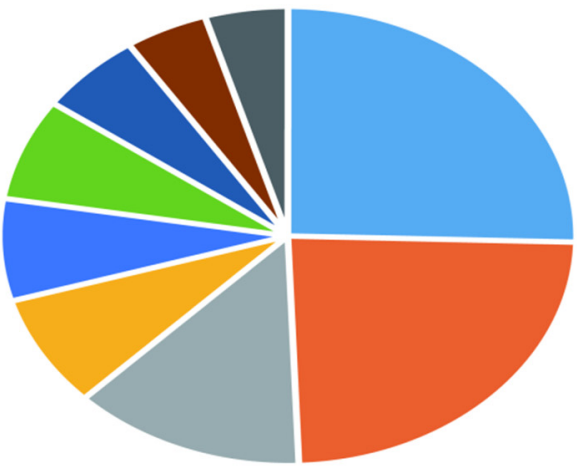

\begin{abstract}
- Computer vision
- Smart medical

- Smart home
\end{abstract}

\begin{abstract}
- Service robot
- Intelligent driving

- Smart education
\end{abstract}

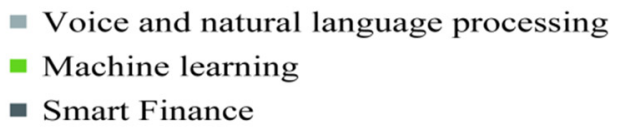

country that implements autonomous remote planning and control. In the application, it is mainly to link the remote intelligent program with the mission objective in the ground system, and then make autonomous planning for the spacecraft. In addition, dynamic real-time monitoring of spacecraft can be realized, and the actual operation of the spacecraft can be more comprehensively and accurately analyzed. In addition, timely detection of operational problems can be made to promptly issue adjustment commands to control the spacecraft, and ultimately ensure the safety and stability of the spacecraft and provide ground researchers with accurate information data references.

\subsection{Robotics}

The application of the artificial intelligence robot field is the most extensive and the earliest. The robot technology emerged in the 1970s, and has since become a professional discipline applied in many fields. Robotic assistants, for example, are used in the medical field to help surgeons perform surgical procedures. The reason that the scope of artificial intelligence applied in the field of robots is constantly expanding. Apart from technical improvements, it is also related to robot artificial intelligence recognition technology. This technology can greatly reduce costs, and it can also protect internal and external risks. From the perspective of the development trend of China's artificial intelligence entrepreneurial enterprises, the research on service robots has become more in-depth. Service robots have appeared in many stores, restaurants, exhibitions, and other places to provide people with services such as navigation, shopping guides, and explanations. As robots in life are still relatively rare, the technology still needs continuous improvement and improvement.

\subsection{Expert System and Intelligent Decision System}

Artificial intelligence began to replace some of human

work with its huge database, even the memory of human spacecrafts. The United States is also the first to use artificial intelligence technology to space outer space. A
Remote planning and control refers to the use of artificial intelligence technology to achieve the planning and control of space vehicles in outer space. For example, NASA of the United States uses computer intelligence programs to achieve precise control and planning of 
beings, so it began to move toward the field of expert systems and intelligent decision systems. In the application, human experience and diagnosis can be analyzed through certain rules and it can made reasonable judgments[4]. The main use is the database of artificial intelligence and then search for something that is related to human memory and form a corresponding pattern. At present, the expert system has been widely used in enterprise management and can quickly formulate corresponding production, scheduling and transportation plans, thereby improving the management level of enterprises, reducing costs and increasing economic benefits.

\subsection{Applied to Auto Control}

Computer vision has always been the main development direction of artificial intelligence entrepreneurial enterprises.With the continuous development of computer vision systems, artificial intelligence technology has also been applied to the automotive field. Through the installation of computer vision systems in automobiles, autonomous control of automobiles can be realized. The car runs steadily on the driveway. At present, the United States has applied this technology to micro-cars to complete autonomous navigation and autonomous driving control through artificial intelligence. However, the application of this field is still only a preliminary stage of exploration. In the future, it is necessary to allow the visual system to accumulate more human application experience, control the direction of the car, and calculate the best driving direction. In the future, driverless cars will become a research hotspot, which is one of the most important applications of artificial intelligence.

\section{Artificial Intelligence Future Trends}

At present, China is in an important period of economic transformation. The manufacturing industry is facing the problem of industrial upgrading. According to "Made in China 2025", it is clearly pointed out that intelligent manufacturing and high-end technological innovation should be regarded as key construction projects, and in particular service robots and other artificial intelligence technologies should be We will give priority to the development of technologies in the future to cultivate artificial intelligence core enterprises. From the current research hotspot of artificial intelligence, it mainly focuses on data mining, knowledge realization, and intelligent interface technologies. Therefore, the development of artificial intelligence in the future may be focused on fuzzy processing technology, parallelization, neural networks, and machine emotions. There will be a breakthrough in technology, wider application areas, and more attention to the ethical issues of artificial intelligence.

\subsection{Advanced Technology and Wider Application Area}

In technology, more attention will be paid to the research of artificial neural networks. Although artificial neural networks can never replace human brains, the processing of information through neuron interactions and the storage of knowledge and information can help humans broaden their knowledge and conduct intelligent control. In the future, the development trend of research on artificial neural networks mainly presents three characteristics: firstly, the artificial neural network model has a fast generation efficiency; secondly, the artificial neural network model is continuously improved and improved; in addition, the artificial neural network is integrated with other calculation methods and applications, such as the combination of neural networks and genetics, and the combination of chaos theory [5].

Although the development of computer technology tends to be gentle at this stage, and integration with other disciplines and fields is also continuing to slow down, artificial intelligence based on computer technology will enter a period of gentle development. However, in the future, artificial intelligence technology will continue to deepen with the development of computer technology, and some relatively low-level artificial intelligence technologies will develop rapidly. However, due to the fact that many fields have not yet reached full integration with other disciplines in applications, there is also great room for development in the future. In addition, in terms of applications, artificial intelligence technology can't really realize the smart life of smart homes under the existing computer technology conditions, and life intelligence is still in an exploratory stage, so in the future, artificial intelligence technology will move towards people's lives. Closely linked to the development of the direction, the real intelligent robots may approach millions of households in the future, and have a great impact on human daily life. Of course, in the fields of transportation and medical care, the development of artificial intelligence technology will also promote the realization of intelligent transportation and intelligent medical care, and improve the efficiency of work in two areas through fully automated methods.

\subsection{More Attention to the Ethical Issues of Artificial Intelligence}

Artificial intelligence will increasingly focus on robot emotions in the future development. That is to say, how do intelligent robots "communicate" with each other and get along with humans after they enter human life? Does artificial intelligence have the same thinking as humans, and will human minds be replaced by robots? These issues will be the focus of future research, especially with the continuous progress of artificial intelligence technology, ethical issues will become a hot topic. Regarding the ethical issues of robots, whether robots should have the same emotions and rights as humans will become the focus of every expert, scholars and scientists, and even every member of society.

\subsection{Notice Things}


With technological breakthroughs in artificial intelligence and thinking about robot emotions, we should pay attention to the following points when we study artificial intelligence technologies in the future: First, we should adhere to the Marxist philosophy of philosophy, that is, we must adhere to the concept of people-oriented, and develop people. The initiative and the role of the main body must not be misplaced in that robots can replace all human activities and even thinking; secondly, they must continuously improve the scientific knowledge and literacy of the public, such as the popularization of scientific and cultural knowledge among the masses, and the promotion of artificial intelligence technology. There is a deep understanding of development so that mistakes can be avoided. In addition, it is necessary to establish the development standards of artificial intelligence and make reasonable plans for the future development and application of artificial intelligence technologies.

\section{Conclusion}

In summary, artificial intelligence technology has already entered people's life and production, and there are more and more industries related to artificial intelligence. The market scale has gradually expanded, bringing great convenience to people's life and production, and has been obtained in many fields. A wide range of applications. In the future, with the deepening of computer technology, artificial intelligence will achieve a greater breakthrough in technology. Research on neural networks, life intelligence, intelligent robots, intelligent transportation, and intelligent medical care will become a hot topic. However, in its development, people must realize that artificial intelligence is by no means omnipotent and will never replace human beings as a dominant position. Therefore, it is also necessary to study its ethical morality and not to influence correct human values due to the ethical issues of robots. This paper conducts some research and analysis on the application and development of computer artificial intelligence technology. It is concluded that in the future development, we must pay more attention to the development of its technology and application. At the same time, we must pay more attention to the ethical issues and make the development of artificial intelligence technology a human being. Development provides a safe and stable technological environment.

\section{References:}

1. Luo Yong, Xiang Yixue. Research Progress and Application of Computer Artificial Intelligence Technology[J]. Electronic production2014(18).

2. Wang Jia. Application bottleneck of computer artificial intelligence identification technology [J]. Science and Technology Outlook, 2016 (35).

3. Shi Ruifang. Application of Artificial Intelligence in Computer Network Technology[J]. Communications World, 2016(23).
4. Tan Shiping. Application Analysis of Artificial Intelligence in Computer Network Technology[J]. Silicon Valley, 2013(18): 11+4.

5. Yang Heng. Research progress and application of computer artificial intelligence technology[J]. Information and Communications, 2014(01): 130. 